\title{
On Coxeter recurrences
}

\author{
Anna Cima, Armengol Gasull and Francesc Mañosas \\ Dept. de Matemàtiques, Facultat de Ciències, \\ Universitat Autònoma de Barcelona, \\ 08193 Bellaterra, Barcelona, Spain \\ cima@mat.uab.cat,gasull@mat.uab.cat,manyosas@mat.uab.cat
}

\begin{abstract}
An interesting family of recurrences of order $n \geq 2$ which are globally $(n+3)$-periodic was introduced by Coxeter in 1971. We prove a surprising property of this family: "all" the possible geometrical behaviors that linear real $(n+3)$-periodic recurrences can have are present inside the Coxeter recurrences.
\end{abstract}

2000 Mathematics Subject Classification: 39A05, 39A20, 39B12.

Keywords: Globally periodic difference equations, recurrences, Coxeter difference equations, linearization.

\section{Introduction and main result}

Although recently globally periodic recurrences have attracted the interest of many researchers, see for instance $[1,2,3,4,5,7,8]$ and the references there in, an interesting family introduced by Coxeter in 1971, see [6], is rarely referenced.

For each natural number $n \geq 2$, Coxeter proved that the recurrences

$$
x_{j+n}=1-\frac{x_{j+n-1}}{1-\frac{x_{j+n-2}}{1-\frac{x_{j+n-3}}{1-\cdots \frac{x_{j+1}}{1-x_{j}}}}}:=f_{n}\left(x_{j}, x_{j+1}, \ldots, x_{j+n-1}\right),
$$

are globally $(n+3)$-periodic, that is for any admissible set of initial conditions, $x_{j+n+3}=x_{j}$, for all $j \geq 0$. For instance, for $n=2,3$, the recurrences are

$$
x_{j+2}=1-\frac{x_{j+1}}{1-x_{j}}, \quad \text { and } \quad x_{j+3}=1-\frac{x_{j+2}}{1-\frac{x_{j+1}}{1-x_{j}}}=\frac{1-x_{j}-x_{j+1}-x_{j+2}+x_{j} x_{j+2}}{1-x_{j}-x_{j+1}},
$$

respectively. It is easy to see that for $n=2$, in the new variables $u_{j}=x_{j}-1$, it corresponds to the well-known 5-periodic Lyness recurrence

$$
u_{j+2}=\frac{1+u_{j+1}}{u_{j}} .
$$


As usual, to study the recurrence (1) we will consider the discrete dynamical system given by the map

$$
F_{n}\left(x_{1}, x_{2}, \ldots, x_{n}\right)=\left(x_{2}, x_{3}, \ldots, x_{n}, f_{n}\left(x_{1}, x_{2}, \ldots, x_{n}\right)\right) .
$$

In his paper Coxeter gives a proof that these recurrences are globally $(n+3)$-periodic, based on the properties of some cross-ratios. Here we will give a new algebraic proof of this fact. As we will see, this new approach will also allow us to prove our main result. It shows the surprising fact that "all" the possible geometrical behaviors that linear real globally periodic recurrences can have are present inside to Coxeter map.

In the next result, as usual, $[s]$ denotes the integer part of $s$.

Theorem. The Coxeter difference equations, given by the maps $F_{n}$ in (2), are globally $(n+3)$-periodic and they have exactly $\left[\frac{n+2}{2}\right]$ fixed points, all of them with positive coordinates. At each of these fixed points $F_{n}$ is locally conjugated to a linear $(n+3)$-periodic recurrence which has no line of fixed points. Moreover, all these $n+3$ linear maps are not conjugated among them. As a consequence, all linear globally $(n+3)$-periodic recurrences having no line of fixed points are present in the Coxeter difference equation.

\section{Preliminary results}

The following classical result will be used to prove that the maps $F_{n}$ linearize in a neighborhood of each fixed point.

Theorem 1. (Montgomery-Bochner Theorem, see [10]). Let $\mathcal{U} \subset \mathbb{R}^{n}$ be an open set and let $F: \mathcal{U} \rightarrow \mathcal{U}$ be a map of class $\mathcal{C}^{r}(\mathcal{U}), r \geq 1$, such that $F^{m}=I d$ for some integer number $m \geq 1$. If $p \in \mathcal{U}$ is a fixed point of $F$ then there is a neighborhood of $p$ in $\mathcal{U}$ where the dynamical system generated by $F$ is $\mathcal{C}^{r}$ linearizable. Moreover the conjugated linear system is given by the linear map $L(x):=d(F)_{p} x$.

The next lemma counts the number of real linear globally $(n+3)$-periodic recurrences. A concrete example is given in Subsection 3.1.

Lemma 2. There are $2\left[\frac{n+2}{2}\right]$ different types of globally $(n+3)$-periodic real linear recurrences of order $n$ when $n$ is odd and $\left[\frac{n+2}{2}\right]$ types when $n$ is even. Moreover there are only $\left[\frac{n+2}{2}\right]$ of them without the eigenvalue 1 .

Proof. Let $L: \mathbb{R}^{n} \rightarrow \mathbb{R}^{n}$ be the globally periodic linear map

$$
L\left(x_{1}, \ldots, x_{n}\right)=\left(x_{2}, \ldots, x_{n}, a_{1} x_{1}+a_{2} x_{2}+\cdots+a_{n} x_{n}\right)
$$

associated to the periodic recurrence. It is known that the characteristic polynomial of $L$ has not multiple roots, see [7,9]. On the other hand it is a real polynomial of degree $n$ and 
all its roots also must be $(n+3)$-roots of the unity. So it divides $\lambda^{n+3}-1$. Thus the proof follows by counting the different number of possibilities for removing a degree 3 real factor from $\lambda^{n+3}-1$.

The following result collects several properties of the rational maps $f_{n}$. Note that $f_{n}(0, \ldots, 0)=1$. So we can write $f_{n}=P_{n} / Q_{n}$ where $P_{n}, Q_{n} \in \mathbb{R}\left[x_{1}, \ldots, x_{n}\right], \operatorname{gcd}\left(P_{n}, Q_{n}\right)=$ 1 and $P_{n}(0)=Q_{n}(0)=1$.

Proposition 3. The polynomials $P_{n}$ and $Q_{n}$ satisfy the following properties:

(i) $P_{n}\left(x_{1}, \ldots, x_{n}\right)=P_{n-1}\left(x_{1}, \ldots, x_{n-1}\right)-x_{n} P_{n-2}\left(x_{1}, \ldots, x_{n-2}\right)$.

(ii) $Q_{n}=P_{n-1}$.

(iii) $P_{n}\left(x_{1}, \ldots, x_{n}\right)=1-\sum_{i=1}^{n} x_{i}+(-1)^{2} \sum_{\substack{j_{1}<j_{2} \leq n \\ j_{2} \neq j_{1}+1}} x_{j_{1}} x_{j_{2}}+\ldots+(-1)^{k_{n}} \sum_{\substack{j_{1}<j_{2}<\ldots<j_{k_{n}} \leq n \\ j_{i+1} \neq j_{i}+1}} x_{j_{1}} \ldots x_{j_{k_{n}}}$, where $k_{n}=\operatorname{deg}\left(P_{n}\right)=\left[\frac{n+1}{2}\right]$.

(iv) $P_{n}\left(x_{1}, x_{2}, \ldots, x_{n-1}, x_{n}\right)=P_{n}\left(x_{n}, x_{n-1}, \ldots, x_{2}, x_{1}\right)$.

(v) $P_{n}\left(x_{1}, \ldots, x_{n}\right)=P_{n-1}\left(x_{2}, \ldots, x_{n}\right)-x_{1} P_{n-2}\left(x_{3}, \ldots, x_{n}\right)$.

Proof. Notice that the maps $f_{n}: \mathbb{R}^{n} \rightarrow \mathbb{R}$ that define the Coxeter difference equations can be given recursively as $f_{1}(x)=1-x$ and

$$
f_{n}\left(x_{1}, x_{2}, \ldots, x_{n}\right)=1-\frac{x_{n}}{f_{n-1}\left(x_{1}, x_{2}, \ldots, x_{n-1}\right)}, \quad n \geq 2 .
$$

We have

$$
\begin{aligned}
\frac{P_{n}}{Q_{n}}\left(x_{1}, \ldots, x_{n}\right) & =f_{n}\left(x_{1}, \ldots, x_{n}\right)=1-\frac{x_{n}}{f_{n-1}\left(x_{1}, \ldots, x_{n-1}\right)}=1-\frac{x_{n}}{\frac{P_{n-1}}{Q_{n-1}}\left(x_{1}, \ldots, x_{n-1}\right)} \\
& =\frac{P_{n-1}\left(x_{1}, \ldots, x_{n-1}\right)-x_{n} Q_{n-1}\left(x_{1}, \ldots, x_{n-1}\right)}{P_{n-1}\left(x_{1}, \ldots, x_{n-1}\right)} .
\end{aligned}
$$

Since $\operatorname{gcd}\left(P_{n-1}-x_{n} Q_{n-1}, P_{n-1}\right)=\operatorname{gcd}\left(Q_{n-1}, P_{n-1}\right)=1$ and $\left(P_{n-1}-x_{n} Q_{n-1}\right)(0)=$ $P_{n-1}(0)=1$, statements (i) and (ii) follow.

Now we prove (iii) by induction. Since $P_{1}\left(x_{1}\right)=1-x_{1}$ and $P_{2}\left(x_{1}, x_{2}\right)=1-x_{1}-x_{2}$, item (iii) is true for $n=1,2$. Now assume that (iii) holds for any $i<n$. Then from (i) we 
have that

$$
\begin{aligned}
& P_{n}\left(x_{1}, \ldots, x_{n}\right)=P_{n-1}\left(x_{1}, \ldots, x_{n-1}\right)-x_{n} P_{n-2}\left(x_{1}, \ldots, x_{n-2}\right) \\
& =1-\sum_{i=1}^{n-1} x_{i}+(-1)^{2} \sum_{\substack{j_{1}<j_{2} \leq n-1 \\
j_{2} \neq j_{1}+1}} x_{j_{1}} x_{j_{2}}+\ldots+(-1)^{k_{n-1}} \sum_{\substack{j_{1}<j_{2}<\ldots<j_{k_{n-1}} \leq n-1 \\
j_{i+1} \neq j_{i}+1}} x_{j_{1}} \ldots x_{j_{k_{n-1}}} \\
& -x_{n}\left(1-\sum_{i=1}^{n-2} x_{i}+(-1)^{2} \sum_{\substack{j_{1}<j_{2} \leq n-2 \\
j_{2} \neq j_{1}+1}} x_{j_{1}} x_{j_{2}}+\ldots+(-1)^{k_{n-2}} \sum_{\substack{j_{1}<j_{2}<\ldots<j_{k_{n-2}} \leq n-2 \\
j_{i+1} \neq j_{i}+1}} x_{j_{1}} \ldots x_{j_{k_{n-2}}}\right) .
\end{aligned}
$$

If we denote by $P_{n}^{l}$ the homogeneous part of degree $l$ of $P_{n}$ we will get

$$
\begin{aligned}
P_{n}^{l}\left(x_{1}, \ldots, x_{n}\right) & =(-1)^{l} \sum_{\substack{j_{1}<j_{2}<\ldots<j_{l} \leq n-1 \\
j_{i+1} \neq j_{i}+1}} x_{j_{1}} \ldots x_{j_{l}}-x_{n}\left((-1)^{l-1} \sum_{\substack{j_{1}<j_{2}<\ldots<j_{l-1} \leq n-2 \\
j_{i+1} \neq j_{i}+1}} x_{j_{1}} \ldots x_{j_{l-1}}\right) \\
& =(-1)^{l} \sum_{\substack{j_{1}<j_{2}<\ldots<j_{l} \leq n \\
j_{i+1} \neq j_{i}+1}} x_{j_{1}} \ldots x_{j_{l}}
\end{aligned}
$$

as we want to prove.

Now (iv) is a direct consequence of (iii), and item (v) is obtained by using (i) and (iv).

\section{Proof of the Theorem}

To prove the periodicity of $F_{n}$ we compute the iterates of $F_{n}$. To do this we will denote by $f_{n}^{i}\left(x_{1}, \ldots, x_{n}\right)=f_{n}\left(F_{n}^{i-1}\left(x_{1}, \ldots, x_{n}\right)\right)$. With this notation $f_{n}^{1}\left(x_{1}, \ldots, x_{n}\right)=f_{n}\left(x_{1}, \ldots, x_{n}\right)=$ $\frac{P_{n}\left(x_{1}, \ldots, x_{n}\right)}{P_{n-1}\left(x_{1}, \ldots, x_{n-1}\right)}$. Now we claim that:

(a) $f_{n}^{2}\left(x_{1}, \ldots, x_{n}\right)=\frac{\prod_{i=1}^{n} x_{i}}{P_{n-1}\left(x_{1}, \ldots, x_{n-1}\right) P_{n-1}\left(x_{2}, \ldots, x_{n}\right)}$ and

$$
P_{n}\left(x_{2}, \ldots, x_{n}, \frac{P_{n}}{P_{n-1}}\left(x_{1}, \ldots, x_{n}\right)\right)=\frac{\prod_{i=1}^{n} x_{i}}{P_{n-1}\left(x_{1}, \ldots, x_{n-1}\right)} .
$$

(b) $f_{n}^{3}\left(x_{1}, \ldots, x_{n}\right)=\frac{P_{n}\left(x_{1}, \ldots, x_{n}\right)}{P_{n-1}\left(x_{2}, \ldots, x_{n}\right)}$ and

$$
P_{n-1}\left(x_{3}, \ldots, \frac{P_{n}}{P_{n-1}}\left(x_{1}, \ldots, x_{n}\right)\right)=\frac{x_{2} \ldots x_{n}}{P_{n-1}\left(x_{1}, \ldots, x_{n-1}\right)} .
$$

(c) $f_{n}^{3+i}\left(x_{1}, \ldots, x_{n}\right)=x_{i}$ for $i=1, \ldots, n$.

To prove (a) it suffices to show that

$$
\frac{P_{n}}{P_{n-1}}\left(F_{n}\left(x_{1}, \ldots, x_{n}\right)\right)=\frac{\prod_{i=1}^{n} x_{i}}{P_{n-1}\left(x_{1}, \ldots, x_{n-1}\right) P_{n-1}\left(x_{2}, \ldots, x_{n}\right)} .
$$


Clearly this last equality is equivalent to

$$
P_{n}\left(x_{2}, \ldots, x_{n}, \frac{P_{n}}{P_{n-1}}\left(x_{1}, \ldots, x_{n}\right)\right)=\frac{\prod_{i=1}^{n} x_{i}}{P_{n-1}\left(x_{1}, \ldots, x_{n-1}\right)},
$$

and using Proposition 3(i) this last equality is also equivalent to

$$
P_{n-1}\left(x_{2}, \ldots, x_{n}\right) P_{n-1}\left(x_{1}, \ldots, x_{n-1}\right)-P_{n}\left(x_{1}, \ldots, x_{n}\right) P_{n-2}\left(x_{2}, \ldots, x_{n-1}\right)=\prod_{i=1}^{n} x_{i} .
$$

Now we prove by induction that equation (3) holds. Easy computations show that (3) holds for $n=3$. So we assume that it holds for $i<n$. We get

$$
P_{n-2}\left(x_{2}, \ldots, x_{n-1}\right) P_{n-2}\left(x_{1}, \ldots, x_{n-2}\right)-P_{n-1}\left(x_{1}, \ldots, x_{n-1}\right) P_{n-3}\left(x_{2}, \ldots, x_{n-2}\right)=\prod_{i=1}^{n-1} x_{i}
$$

By multiplying this equality by $x_{n}$ we have

$x_{n} P_{n-2}\left(x_{2}, \ldots, x_{n-1}\right) P_{n-2}\left(x_{1}, \ldots, x_{n-2}\right)-x_{n} P_{n-1}\left(x_{1}, \ldots, x_{n-1}\right) P_{n-3}\left(x_{2}, \ldots, x_{n-2}\right)=\prod_{i=1}^{n} x_{i}$.

From Proposition 3(i) we have that

$$
x_{n} P_{n-2}\left(x_{1}, \ldots, x_{n-2}\right)=P_{n-1}\left(x_{1}, \ldots, x_{n-1}\right)-P_{n}\left(x_{1}, \ldots, x_{n}\right) .
$$

Substituting this last equality in the previous equation we obtain

$$
\begin{aligned}
\prod_{i=1}^{n} x_{i} & =P_{n-2}\left(x_{2}, \ldots, x_{n-1}\right)\left(P_{n-1}\left(x_{1}, \ldots, x_{n-1}\right)-P_{n}\left(x_{1}, \ldots, x_{n}\right)\right) \\
& -x_{n} P_{n-1}\left(x_{1}, \ldots, x_{n-1}\right) P_{n-3}\left(x_{2}, \ldots, x_{n-2}\right) \\
& =P_{n-1}\left(x_{1}, \ldots, x_{n-1}\right)\left(P_{n-2}\left(x_{2}, \ldots, x_{n-1}\right)-x_{n} P_{n-3}\left(x_{2}, \ldots, x_{n-2}\right)\right) \\
& -P_{n-2}\left(x_{2}, \ldots, x_{n-1}\right) P_{n}\left(x_{1}, \ldots, x_{n}\right) \\
& =P_{n-1}\left(x_{2}, \ldots, x_{n}\right) P_{n-1}\left(x_{1}, \ldots, x_{n-1}\right)-P_{n}\left(x_{1}, \ldots, x_{n}\right) P_{n-2}\left(x_{2}, \ldots, x_{n-1}\right)
\end{aligned}
$$

as we want to prove.

Now we prove (b). Since $f_{n}^{3}\left(x_{1}, \ldots, x_{n}\right)=f_{n}\left(F_{n}^{2}\left(x_{1}, \ldots, x_{n}\right)\right)=f_{n}^{2}\left(F_{n}\left(x_{1}, \ldots, x_{n}\right)\right)$ using statement (a) applied to the point $F\left(x_{1}, \ldots, x_{n}\right)$ instead of $\left(x_{1}, \ldots, x_{n}\right)$ we obtain

$$
\begin{aligned}
f_{n}^{3}\left(x_{1}, \ldots, x_{n}\right) & =f_{n}^{2}\left(x_{2}, \ldots, x_{n}, \frac{P_{n}}{P_{n-1}}\left(x_{1}, \ldots, x_{n}\right)\right) \\
& =\frac{x_{2} \ldots x_{n} \frac{P_{n}}{P_{n-1}}\left(x_{1}, \ldots, x_{n}\right)}{P_{n-1}\left(x_{2}, \ldots, x_{n}\right) P_{n-1}\left(x_{3}, \ldots, x_{n}, \frac{P_{n}}{P_{n-1}}\left(x_{1}, \ldots, x_{n}\right)\right)} .
\end{aligned}
$$

Thus it suffices to show that

$$
P_{n-1}\left(x_{1}, \ldots, x_{n-1}\right) P_{n-1}\left(x_{3}, \ldots, x_{n}, \frac{P_{n}}{P_{n-1}}\left(x_{1}, \ldots, x_{n}\right)\right)=x_{2} \ldots x_{n},
$$


which is the equality claimed in statement (b). From Proposition 3(i) it is equivalent to

$$
P_{n-1}\left(x_{1}, \ldots, x_{n-1}\right) P_{n-2}\left(x_{3}, \ldots, x_{n}\right)-P_{n-3}\left(x_{3}, \ldots, x_{n-1}\right) P_{n}\left(x_{1}, \ldots, x_{n}\right)=x_{2} \ldots x_{n} .
$$

Also from Proposition 3(v) we have that

$$
P_{n-1}\left(x_{1}, \ldots, x_{n-1}\right)=P_{n-2}\left(x_{2}, \ldots, x_{n-1}\right)-x_{1} P_{n-3}\left(x_{3}, \ldots, x_{n-1}\right)
$$

and

$$
P_{n}\left(x_{1}, \ldots, x_{n}\right)=P_{n-1}\left(x_{2}, \ldots, x_{n}\right)-x_{1} P_{n-2}\left(x_{3}, \ldots, x_{n}\right) .
$$

Substituting these last equalities in the previous one we obtain

$$
P_{n-2}\left(x_{2}, \ldots, x_{n-1}\right) P_{n-2}\left(x_{3}, \ldots, x_{n}\right)-P_{n-1}\left(x_{2}, \ldots, x_{n}\right) P_{n-3}\left(x_{3}, \ldots, x_{n-1}\right)=x_{2} \ldots x_{n},
$$

which holds because it is equation (4) for $\left(x_{2}, \ldots, x_{n}\right)$ instead of $\left(x_{1}, \ldots, x_{n-1}\right)$. Thus (b) is satisfied.

Now we prove (c). For $i=1$ we have

$$
f_{n}^{4}\left(x_{1}, \ldots, x_{n}\right)=f_{n}^{3}\left(x_{2}, \ldots, \frac{P_{n}}{P_{n-1}}\left(x_{1}, \ldots, x_{n}\right)\right)=\frac{P_{n}\left(x_{2}, \ldots, \frac{P_{n}}{P_{n-1}}\left(x_{1}, \ldots, x_{n}\right)\right)}{P_{n-1}\left(x_{3}, \ldots, \frac{P_{n}}{P_{n-1}}\left(x_{1}, \ldots, x_{n}\right)\right)} .
$$

Now using statements (a) and (b) we obtain

$$
f_{n}^{4}\left(x_{1}, \ldots, x_{n}\right)=\frac{\frac{\prod_{i=1}^{n} x_{i}}{P_{n-1}\left(x_{1}, \ldots, x_{n-1}\right)}}{\frac{x_{2} \ldots x_{n}}{P_{n-1}\left(x_{1}, \ldots, x_{n-1}\right)}}=x_{1} .
$$

Set $i$ such that $1<i \leq n$. Then we will have

$$
f_{n}^{3+i}\left(x_{1}, \ldots, x_{n}\right)=f_{n}^{4}\left(F^{i-1}\left(x_{1}, \ldots, x_{n}\right)\right)=f_{n}^{4}\left(x_{i}, \ldots\right)=x_{i} .
$$

This ends the proof of the claim.

Then since $F^{n+3}\left(x_{1}, \ldots, x_{n}\right)=\left(f_{n}^{4}\left(x_{1}, \ldots, x_{n}\right), \ldots, f_{n}^{n+3}\left(x_{1}, \ldots, x_{n}\right)\right)=\left(x_{1}, \ldots, x_{n}\right)$ we have proved that $F_{n}$ is globally $(n+3)$-periodic.

Now we study the fixed points of $F_{n}$. Since $F_{n}$ is a recurrence all fixed points are of the form $(x, \ldots, x)$ and must satisfy $P_{n-1}(x, \ldots, x) \neq 0$ and $\frac{P_{n}}{P_{n-1}}(x, \ldots, x)=x$. We introduce the following notation. For any $i \in \mathbb{N}$ and for any $x \in \mathbb{R}$ we denote by $p_{i}(x):=P_{i}(x, \ldots, x)$ where $(x, \ldots, x) \in \mathbb{R}^{i}$. Observe that from Proposition 3.(i) we obtain

$$
p_{k+1}(x)=p_{k}(x)-x p_{k-1}(x)
$$


for all $k \in \mathbb{N}$. With this notation the fixed points of $F_{n}$ are the points $(x, \ldots, x) \in \mathbb{R}^{n}$ satisfying $p_{n+1}(x)=0$ and $p_{n-1}(x) \neq 0$. First of all we note that $p_{n+1}(x)$ and $p_{n-1}(x)$ have not common zeros because if $p_{n+1}(x)=p_{n-1}(x)=0$ then from equation (5) we get that $p_{n}(x)=0$ and applying recursively this result we get that $1-x=p_{1}(x)=p_{2}(x)=$ $1-2 x=0$, which gives a contradiction. Then the fixed points of $F_{n}$ are precisely the points $(x, \ldots, x) \in \mathbb{R}^{n}$ such that $x$ is a zero of $p_{n+1}(x)$. Recall that from Proposition 3(iii) we have that $d(n):=\operatorname{deg}\left(p_{n+1}\right)=\left[\frac{n+2}{2}\right]$. Now we claim that $p_{n+1}(x)$ has exactly $d(n)$ positive roots and we will prove this assertion by induction. From Proposition 3(iii) we know that

$$
p_{n+1}(x)=1+\cdots+c_{d(n)} x^{d(n)} \quad \text { with } \quad d(n) \neq 0 .
$$

Denote by $x_{n, 1} \leq x_{n, 2} \leq \ldots \leq x_{n, j}, j \leq d(n)$, all the real roots of $p_{n+1}(x)$. These are our hypotheses of induction:

- When $n \geq 2$ is even, $p_{n+1}$ and $p_{n+2}$ have the same degree, $d(n)$, and all their roots are real, simple and positive. Moreover all of them are intercalated and satisfy:

$$
0<x_{n+1,1}<x_{n, 1}<x_{n+1,2}<x_{n, 2}<\ldots<x_{n, d(n)-1}<x_{n+1, d(n)}<x_{n, d(n)} .
$$

- When $n \geq 1$ is odd, $p_{n+1}$ and $p_{n+2}$ have degree $d(n)$ and $d(n+1)=d(n)+1$, respectively, and all their roots are real and positive. Moreover all of them are real, simple and intercalated and satisfy:

$$
0<x_{n+1,1}<x_{n, 1}<x_{n+1,2}<x_{n, 2}<\ldots<x_{n+1, d(n)}<x_{n, d(n)}<x_{n+1, d(n)+1} .
$$

The first polynomials are $p_{2}(x)=1-2 x, p_{3}(x)=1-3 x+x^{2}, p_{4}(x)=1-4 x+3 x^{2}$ and $p_{5}(x)=1-5 x+6 x^{2}-x^{3}$ and it is easy to see that they satisfy our induction hypotheses.

Consider, for instance the case $n$ even and let us see how knowing that the roots of $p_{n+1}$ and $p_{n+2}$ satisfy the induction hypotheses, the same holds with the roots of $p_{n+2}$ and $p_{n+3}$. Since $p_{n+3}(0)=p_{n+2}(0)=p_{n+1}(0)=1$, by using equation (5) and the induction hypotheses we know that

$$
p_{n+3}\left(x_{n+1,1}\right)=p_{n+2}\left(x_{n+1,1}\right)-x_{n+1,1} p_{n+1}\left(x_{n+1,1}\right)=-x_{n+1,1} p_{n+1}\left(x_{n+1,1}\right)<0 .
$$

Hence $0<x_{n+2,1}<x_{n+1,1}$, as we wanted to show. By using again equation (5) and taking into account that all the roots of $p_{n+1}$ and $p_{n+2}$ are real, simple and intercalated, it is easy to check that $(-1)^{j} p_{n+3}\left(x_{n+1, j}\right)>0$ for $j=2, \ldots, d(n+1)$, proving that $p_{n+3}$ has $d(n)=d(n+1)$ roots, intercalated with the ones of $p_{n+1}$. By studying the behavior of $p_{n+3}$ at infinity we get one more root, bigger than $x_{n+1, d(n+1)}$ as we wanted to see. The case $n$ odd can be studied similarly. 
By the Montgomery-Bochner Theorem (see Theorem 1) we know that the map $F_{n}$ associated to the Coxeter recurrences (1) linearize on a neighborhood of each of its $\left[\frac{n+2}{2}\right]$ fixed points. The fact that these recurrences have a finite number of fixed points prevents the possibility of having 1 as a eigenvalue of the characteristic polynomial associated to each of them. By using Lemma 2 we know that there are only $\left[\frac{n+2}{2}\right]$ different linear recurrences without the eigenvalue 1 . Hence to finish the proof of the Theorem it suffices to show that if $\mathbf{x}=(x, \ldots, x)$ and $\mathbf{y}=(y, \ldots, y)$ are different fixed points of $F_{n}$ then $d\left(F_{n}\right)_{\mathbf{x}}$ and $d\left(F_{n}\right)_{\mathbf{y}}$ are linear models not conjugated. To see this it is enough to prove that $\frac{\partial f_{n}}{\partial x_{n}}(\mathbf{x})=$ $\operatorname{Trace}\left(d\left(F_{n}\right)_{\mathbf{x}}\right) \neq \operatorname{Trace}\left(d\left(F_{n}\right)_{\mathbf{y}}\right)=\frac{\partial f_{n}}{\partial x_{n}}(\mathbf{y})$. By Proposition 3(i) it follows that

$$
\frac{\partial f_{n}}{\partial x_{n}}\left(x_{1}, \ldots, x_{n}\right)=\frac{\partial\left(1-x_{n} \frac{P_{n-2}\left(x_{1}, \ldots, x_{n-2}\right)}{P_{n-1}\left(x_{1}, \ldots, x_{n-1}\right)}\right)}{\partial x_{n}}=-\frac{P_{n-2}\left(x_{1}, \ldots, x_{n-2}\right)}{P_{n-1}\left(x_{1}, \ldots, x_{n-1}\right)} .
$$

In particular we will get $\frac{\partial f_{n}}{\partial x_{n}}(\mathbf{x})=-\frac{p_{n-2}(x)}{p_{n-1}(x)}$ and $\frac{\partial f_{n}}{\partial x_{n}}(\mathbf{y})=-\frac{p_{n-2}(y)}{p_{n-1}(y)}$. On the other hand since $\mathbf{x}$ and $\mathbf{y}$ are fixed points we get that $\frac{p_{n-2}(x)}{p_{n-1}(x)}=\frac{x-1}{x}$ and $\frac{p_{n-2}(y)}{p_{n-1}(y)}=\frac{y-1}{y}$, hence we obtain

$$
\frac{\partial f_{n}}{\partial x_{n}}(\mathbf{x})=\frac{1-x}{x} \neq \frac{1-y}{y}=\frac{\partial f_{n}}{\partial x_{n}}(\mathbf{y}),
$$

as we want to prove.

\subsection{An example: $n=5$}

In this section we illustrate our results for Coxeter maps taking $n=5$. In this case the map (2) writes as

$$
F_{5}\left(x_{1}, x_{2}, x_{3}, x_{4}, x_{5}\right)=\left(x_{2}, x_{3}, x_{4}, x_{5}, f_{5}\left(x_{1}, x_{2}, x_{3}, x_{4}, x_{5}\right)\right)
$$

with

$$
f_{5}\left(x_{1}, x_{2}, x_{3}, x_{4}, x_{5}\right)=\frac{1-\sum_{i=1}^{5} x_{i}+x_{1}\left(x_{3}+x_{4}+x_{5}\right)+x_{2}\left(x_{4}+x_{5}\right)+x_{3} x_{5}\left(1-x_{1}\right)}{1-x_{1}-x_{2}-x_{3}-x_{4}+x_{1} x_{3}+x_{1} x_{4}+x_{2} x_{4}},
$$

and it is globally 8-periodic. Following Lemma 3 we know that there are only three real 8 -periodic linear recurrences of order 5 not having the eigenvalue 1 . Since the polynomial $\lambda^{8}-1$ decomposes as

$$
\lambda^{8}-1=(\lambda-1)(\lambda+1)\left(\lambda^{2}+1\right)\left(\lambda^{2}-\sqrt{2} \lambda+1\right)\left(\lambda^{2}+\sqrt{2} \lambda+1\right),
$$

they are the ones having associated characteristic polynomials:

$$
\begin{aligned}
& p_{1}(\lambda)=(\lambda+1)\left(\lambda^{2}+\sqrt{2} \lambda+1\right)\left(\lambda^{2}-\sqrt{2} \lambda+1\right), \\
& p_{2}(\lambda)=(\lambda+1)\left(\lambda^{2}+1\right)\left(\lambda^{2}+\sqrt{2} \lambda+1\right), \quad \text { and } \\
& p_{3}(\lambda)=(\lambda+1)\left(\lambda^{2}+1\right)\left(\lambda^{2}-\sqrt{2} \lambda+1\right) .
\end{aligned}
$$


On the other hand the map $F$ has three fixed points $\mathbf{x}_{i}:=x_{i}(1,1,1,1,1), i=1,2,3$, with $x_{1}=\frac{1}{2}, x_{2}=1-\frac{\sqrt{2}}{2}$ and $x_{3}=1+\frac{\sqrt{2}}{2}$. It is easy to prove that for each $i, i=1,2,3$, the characteristic polynomial of $d\left(F_{5}\right)$ at the point $\mathbf{x}_{i}$ is exactly the polynomial $p_{i}(\lambda)$, as it is predicted by our Theorem.

\section{Acknowledgements}

The authors are partially supported by a MCYT/FEDER grant number MTM2008-03437 and by a CIRIT grant number 2009SGR 410 .

\section{References}

[1] R. M. Abu-Saris and Q. M. Al-Hassan. On global periodicity of difference equations, J. Math. Anal. Appl. 283 (2003), 468-477.

[2] F. Balibrea and A. Linero. Some new results and open problems on periodicity of difference equations, Iteration theory (ECIT '04), 15-38, Grazer Math. Ber., 350, KarlFranzens-Univ. Graz, Graz, 2006.

[3] A. Cima, A. Gasull and F. Mañosas. On periodic rational difference equations of order $k$, J. Difference Equ. and Appl. 10 (2004), 549-559.

[4] A. Cima, A. Gasull and V. Mañosa. Global periodicity and complete integrability of discrete dynamical systems, J. Difference Equ. Appl. 12 (2006), 697-716.

[5] J. S. Cánovas, A. Linero and G. Soler. A characterization of k-cycles, Nonlinear Anal. 72 (2010), 364-372.

[6] H.S. M. Coxeter. Frieze patterns, Acta Arith. 18 (1971), 297-310.

[7] M. Csörnyei and M. Laczkovich. Some periodic and non-periodic recursions, Monatshefte für Mathematik 132 (2001), 215-236.

[8] M.R.S. Kulenovi and G. Ladas, Dynamics of Second Order Rational Difference Equations: With Open Problems and Conjectures, Chapman \& Hall/CRC, Boca Raton, FL, 2002.

[9] R. P. Kurshan and B. Gopinath. Recursively generated periodic sequences, Canad. J. Math. 26 (1974), 1356-1371.

[10] D. Montgomery and L. Zippin. "Topological Transformation Groups", Interscience, New-York (1955). 